\title{
On approximate inclusion-exclusion
}

\author{
Andreas Klein* Klaus Metsch
}

full screen

close

quit

\begin{abstract}
The inclusion-exclusion formula expresses the size of the union of a family of sets in terms of the sizes of intersections of all subfamilies. In [2] $\mathrm{N}$. Linial and N. Nisan use linear programming to approximate the size of the union when the intersection sizes are known only for certain subfamilies. In this article we use purely combinatorial methods to generalize some of their results. As an application we will construct a contrast optimal $(n-1)$-out-of- $n$ visual cryptography scheme.
\end{abstract}

Keywords: inclusion-exclusion formula, visual cryptography

MSC 2000: 05A20, 68P25

\section{Introduction}

The inclusion-exclusion formula states that

$$
\begin{aligned}
\left|A_{1} \cup A_{2} \cup \cdots \cup A_{n}\right|= & \sum_{i}\left|A_{i}\right|-\sum_{i<j}\left|A_{i} \cap A_{j}\right|+ \\
& \sum_{i<j<k}\left|A_{i} \cap A_{j} \cap A_{k}\right|-\cdots-(-1)^{n}\left|A_{1} \cap \cdots \cap A_{n}\right| .
\end{aligned}
$$

Obviously every term on the right-hand side is needed to determine the size of the union. At this point we can ask whether it is possible to give an approximate inclusion-exclusion formula. More formally we ask:

\footnotetext{
*The research of the author takes place within the project "Linear codes and cryptography" of the Fund for Scientific Research Flanders (FWO-Vlaanderen) (Project nr. G.0317.06), and is supported by the Interuniversitary Attraction Poles Programme-Belgian State-Belgian Science Policy: project P6/26-Bcrypt.
} 
Given integers $m, n$ with $m<n$ and sets $A_{1}, \ldots, A_{n}$ and $B_{1}, \ldots, B_{n}$ where not all $B_{i}$ are empty and where

$$
\left|\bigcap_{i \in S} A_{i}\right|=\left|\bigcap_{i \in S} B_{i}\right|
$$

for every subset $S \subseteq\{1, \ldots, n\}$ such that $|S|<m$, what is the smallest (or largest) possible value for the fraction

$$
\frac{\left|A_{1} \cup \cdots \cup A_{n}\right|}{\left|B_{1} \cup \cdots \cup B_{n}\right|} ?
$$

In [2] N. Linial and N. Nisan use linear programming to reduce this question to questions in approximation theory and in particular to the theory of Chebyshev polynomials. Their bound is nearly optimal for $m \leq \sqrt{n}$, but for larger $m$ the bound gets worse. In this paper we give an exact bound for $m=n-2$ and improve the asymptotic bound for $m=n-d, d$ fixed. The results we find have applications in visual cryptography. More results for small $m$ can be found in [1].

\section{The case $m=n-1$}

We start with the case $m=n-1$. This case was solved in [2] using linear programming methods. Here we present a more elementary purely combinatorial proof. Besides being interesting for itself, the proof is a good warm up for the more difficult case $m=n-2$.

Theorem 2.1 (see [2, Theorem 3]). Let $A_{1}, \ldots, A_{n}$ and $B_{1}, \ldots, B_{n}$ be two collections of sets satisfying

$$
\left|\bigcap_{i \in S} A_{i}\right|=\left|\bigcap_{i \in S} B_{i}\right|
$$

for all proper subsets $S$ of $\{1, \ldots, n\}$. Then

$$
\frac{\left|\bigcup_{i=1}^{n} B_{i}\right|-\left|\bigcup_{i=1}^{n} A_{i}\right|}{\left|\bigcup_{i=1}^{n} B_{i}\right|} \leq \frac{1}{2^{n-1}} .
$$

Proof. We prove by induction on $n$ that the conditions

$$
\left|\bigcap_{i \in S} A_{i}\right|=\left|\bigcap_{i \in S} B_{i}\right|
$$


for all $S \subsetneq\{1, \ldots, n\}$ and the condition

$$
\left|\bigcup_{i=1}^{n} A_{i}\right|+k=\left|\bigcup_{i=1}^{n} B_{i}\right|
$$

with $k>0$ imply that

$$
\left|\bigcup_{i=1}^{n} B_{i}\right| \geq k 2^{n-1}
$$

For $n=1$ this is trivial. Now suppose that the theorem holds for $n$ and let the sets $A_{1}, \ldots, A_{n+1}$ and $B_{1}, \ldots, B_{n+1}$ satisfy

$$
\left|\bigcap_{i \in S} A_{i}\right|=\left|\bigcap_{i \in S} B_{i}\right|
$$

for all $S \subsetneq\{1, \ldots, n+1\}$ and

$$
\left|\bigcup_{i=1}^{n+1} A_{i}\right|+k=\left|\bigcup_{i=1}^{n+1} B_{i}\right|
$$

The collections $A_{i}^{\prime}=A_{i} \backslash A_{n+1}$ and $B_{i}^{\prime}=B_{i} \backslash B_{n+1}$ satisfy $\left|\bigcup_{i=1}^{n} A_{i}^{\prime}\right|+k=$ $\left|\bigcup_{i=1}^{n} B_{i}^{\prime}\right|$ and for every proper subset $S \subsetneq\{1, \ldots, n\}$ we have

$$
\begin{aligned}
\left|\bigcap_{i \in S} A_{i}^{\prime}\right| & =\left|\bigcap_{i \in S} A_{i}\right|-\left|\bigcap_{i \in S} A_{i} \cap A_{n+1}\right| \\
& =\left|\bigcap_{i \in S} B_{i}\right|-\left|\bigcap_{i \in S} B_{i} \cap B_{n+1}\right|=\left|\bigcap_{i \in S} B_{i}^{\prime}\right| .
\end{aligned}
$$

Thus the collections $A_{i}^{\prime}$, $B_{i}^{\prime}$ satisfy the induction hypothesis, i.e. we have $\left|\bigcup_{i=1}^{n} B_{i}^{\prime}\right| \geq$ $k 2^{n-1}$.

On the other hand, we have the collections $A_{i}^{\prime \prime}=A_{i} \cap A_{n+1}$ and $B_{i}^{\prime \prime}=$ $B_{i} \cap B_{n+1}$. Since

$$
\begin{aligned}
\left|\bigcup_{i=1}^{n} A_{i}\right| & =\left|\bigcup_{i=1}^{n} B_{i}\right| \\
\Longleftrightarrow\left|\bigcup_{i=1}^{n} A_{i}^{\prime}\right|+\left|\bigcup_{i=1}^{n} A_{i}^{\prime \prime}\right| & =\left|\bigcup_{i=1}^{n} B_{i}^{\prime}\right|+\left|\bigcup_{i=1}^{n} B_{i}^{\prime \prime}\right|
\end{aligned}
$$

and $\left|\bigcup_{i=1}^{n} A_{i}^{\prime}\right|+k=\left|\bigcup_{i=1}^{n} B_{i}^{\prime}\right|$ we find that the collections $A_{i}^{\prime \prime}$ and $B_{i}^{\prime \prime}$ satisfy the induction hypothesis with $\left|\bigcup_{i=1}^{n} B_{i}^{\prime \prime}\right|+k=\left|\bigcup_{i=1}^{n} A_{i}^{\prime \prime}\right|$. Thus $\left|B_{n+1}\right|=\left|A_{n+1}\right| \geq$ $\left|\bigcup_{i=1}^{n} A_{i}^{\prime \prime}\right| \geq k 2^{n-1}$. This proves

$$
\left|\bigcup_{i=1}^{n+1} B_{i}\right|=\left|\bigcup_{i=1}^{n} B_{i}^{\prime}\right|+\left|B_{n+1}\right| \geq k 2^{n-1}+k 2^{n-1}=k 2^{n}
$$




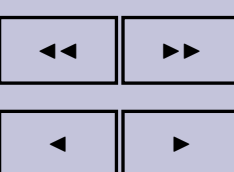

page $5 / 22$

go back

full screen

close

quit

Proof. The proof is very similar to the proof of Theorem 2.1. It is sufficient to consider the case when $k \geq 0$. Suppose $A_{1}, \ldots, A_{n+1}$ and $B_{1}, \ldots, B_{n+1}$ are sets realizing $S_{n+1}(k, x)$, that is (1a), (2a) and (3a) are satisfied and $S_{n+1}(k, x)=$ $\left|\bigcup_{i=1}^{n+1} B_{i}\right|$. Let $y$ be the integer satisfying $\left|\bigcap_{i=1}^{n} A_{i}\right|=\left|\bigcap_{i=1}^{n} B_{i}\right|-(-1)^{n} y$.

The sets $A_{i}^{\prime}=A_{i} \backslash A_{n+1}$ and $B_{i}^{\prime}=B_{i} \backslash B_{n+1}$ satisfy the following conditions:

(1) $\left|\bigcap_{i \in S} A_{i}^{\prime}\right|=\left|\bigcap_{i \in S} B_{i}^{\prime}\right|$ for all $S$ with $|S| \leq n-2$, since

$$
\begin{aligned}
\left|\bigcap_{i \in S} A_{i}^{\prime}\right| & =\left|\bigcap_{i \in S} A_{i}\right|-\left|\bigcap_{i \in S} A_{i} \cap A_{n+1}\right| \\
& =\left|\bigcap_{i \in S} B_{i}\right|-\left|\bigcap_{i \in S} B_{i} \cap B_{n+1}\right|=\left|\bigcap_{i \in S} B_{i}^{\prime}\right| .
\end{aligned}
$$

(2) $\left|\bigcap_{i=1}^{n} A_{i}^{\prime}\right|=\left|\bigcap_{i=1}^{n} B_{i}^{\prime}\right|-(-1)^{n}(y+x)$, since

$$
\begin{aligned}
\left|\bigcap_{i=1}^{n} A_{i}^{\prime}\right| & =\left|\bigcap_{i=1}^{n} A_{i}\right|-\left|\bigcap_{i=1}^{n+1} A_{i}\right| \\
& =\left|\bigcap_{i=1}^{n} B_{i}\right|-(-1)^{n} y-\left[\left|\bigcap_{i=1}^{n+1} B_{i}\right|-(-1)^{n+1} x\right] .
\end{aligned}
$$

(3) $\left|\bigcup_{i=1}^{n} A_{i}^{\prime}\right|+k=\left|\bigcup_{i=1}^{n} B_{i}^{\prime}\right|$, since

$$
\left|\bigcup_{i=1}^{n} A_{i}^{\prime}\right|+\left|A_{n+1}\right|+k=\left|\bigcup_{i=1}^{n+1} A_{i}\right|+k=\left|\bigcup_{i=1}^{n+1} B_{i}\right|=\left|\bigcup_{i=1}^{n} B_{i}^{\prime}\right|+\left|B_{n+1}\right|
$$

and $\left|A_{n+1}\right|=\left|B_{n+1}\right|$.

This proves $\left|\bigcup_{i=1}^{n} B_{i}^{\prime}\right|=\left|\left(\bigcup_{i=1}^{n} B_{i}\right) \backslash B_{n+1}\right| \geq S_{n}(k, x+y)$.

Now we prove a lower bound for $\left|A_{n+1}\right|=\left|B_{n+1}\right|$. The sets $A_{i}^{\prime \prime}=A_{i} \cap A_{n+1}$, $B_{i}^{\prime \prime}=B_{i} \cap B_{n+1}$ satisfy

(1) $\left|\bigcap_{i \in S} A_{i}^{\prime \prime}\right|=\left|\bigcap_{i \in S} B_{i}^{\prime \prime}\right|$ for all $S$ with $|S| \leq n-2$, since $|S|+1 \leq(n+1)-2$ and thus $\left|\bigcap_{i \in S} A_{i}^{\prime \prime}\right|=\left|\bigcap_{i \in S} A_{i} \cap A_{n+1}\right|=\left|\bigcap_{i \in S} B_{i} \cap B_{n+1}\right|=\left|\bigcap_{i \in S} B_{i}^{\prime \prime}\right|$,

(2) $\left|\bigcap_{i=1}^{n} A_{i}^{\prime \prime}\right|-(-1)^{n} x=\left|\bigcap_{i=1}^{n} B_{i}^{\prime \prime}\right|$, since $\bigcap_{i=1}^{n} A_{i}^{\prime \prime}=\bigcap_{i=1}^{n+1} A_{i}$,

(3) $\left|\bigcup_{i=1}^{n} A_{i}^{\prime \prime}\right|=\left|\bigcup_{i=1}^{n} B_{i}^{\prime \prime}\right|+k+y$, since

$$
\begin{aligned}
&\left|\bigcup_{i=1}^{n} A_{i}^{\prime \prime}\right|+\left|\bigcup_{i=1}^{n} A_{i}^{\prime}\right|=\sum_{S, n+1 \notin S}(-1)^{|S|+1}\left|\bigcap_{i \in S} A_{i}\right| \\
&=\sum_{S, n+1 \notin S}(-1)^{|S|+1}\left|\bigcap_{i \in S} B_{i}\right|+y=\left|\bigcup_{i=1}^{n} B_{i}^{\prime \prime}\right|+\left|\bigcup_{i=1}^{n} B_{i}^{\prime}\right|+y .
\end{aligned}
$$




Proof. Without loss of generality we may assume $k \geq 0$.

Part 1: We show $\geq$ in (3).

Consider symmetric configurations $A_{1}, \ldots, A_{n+1}$ and $B_{1}, \ldots, B_{n+1}$ of sets minimizing $\tilde{S}_{n+1}(k, x)$. Using the symmetry and properties (1b), (2b), and (3b) we find

$$
\mu\left(\bigcap_{i=1}^{n} A_{i}\right)=\mu\left(\bigcap_{i=1}^{n} B_{i}\right)+(-1)^{n} \frac{k+x}{n+1} .
$$

full screen

close

quit

Hence the value of $y$ defined in the assertion is the one that was used in the proof of Lemma 3.1. The proof of Lemma 3.1 therefore shows the $\geq$-part of (3).

Part 2: We show $\leq$ in (3).

By induction there exist symmetric collections $A_{1}^{\prime}, \ldots, A_{n}^{\prime}$ and $B_{1}^{\prime}, \ldots, B_{n}^{\prime}$ realizing $\tilde{S}_{n}(k, x+y)$, that is satisfying (1b), (2b) and (3b) and such that $\mu^{\prime}\left(\bigcup_{i=1}^{n} B_{i}^{\prime}\right)=\tilde{S}_{n}(k, x+y)$. There also exist symmetric collections $B_{1}^{\prime \prime}, \ldots, B_{n}^{\prime \prime}$ and $A_{1}^{\prime \prime}, \ldots, A_{n}^{\prime \prime}$ realizing $\tilde{S}_{n}(k+y, x)$. Notice the order of the $B_{i}^{\prime \prime}$ and the $A_{i}^{\prime \prime}$, which is meant as follows:

$$
\begin{aligned}
& \mu^{\prime \prime}\left(\bigcap_{i=1}^{n} B_{i}^{\prime \prime}\right)=\mu^{\prime \prime}\left(\bigcap_{i=1}^{n} A_{i}^{\prime \prime}\right)-(-1)^{n} x, \\
& \mu^{\prime \prime}\left(\bigcup_{i=1}^{n} B_{i}^{\prime \prime}\right)+k+y=\mu^{\prime \prime}\left(\bigcup_{i=1}^{n} A_{i}^{\prime \prime}\right), \\
& \mu^{\prime \prime}\left(\bigcup_{i=1}^{n} B_{i}^{\prime \prime}\right)=\tilde{S}_{n}(k+y, x) \text { if } k+y \leq 0, \text { and } \\
& \mu^{\prime \prime}\left(\bigcup_{i=1}^{n} A_{i}^{\prime \prime}\right)=\tilde{S}_{n}(k+y, x) \text { if } k+y \geq 0 .
\end{aligned}
$$

Without loss of generality we may assume that $\Omega^{\prime}=\bigcup_{i=1}^{n} A_{i}^{\prime} \cup \bigcup_{i=1}^{n} B_{i}^{\prime}$ and $\Omega^{\prime \prime}=\bigcup_{i=1}^{n} A_{i}^{\prime \prime} \cup \bigcup_{i=1}^{n} B_{i}^{\prime \prime}$ are disjoint. The measures $\mu^{\prime}$ on $\Omega^{\prime}$ and $\mu^{\prime \prime}$ on $\Omega^{\prime \prime}$ induce a measure $\mu$ on $\Omega^{\prime} \cup \Omega^{\prime \prime}$.

Define $A_{i}=A_{i}^{\prime} \cup A_{i}^{\prime \prime}$ and $B_{i}=B_{i}^{\prime} \cup B_{i}^{\prime \prime}, i=1, \ldots, n, A_{n+1}=\bigcup_{i=1}^{n} A_{i}^{\prime \prime} \cup A$, and $B_{n+1}=\bigcup_{i=1}^{n} B_{i}^{\prime \prime} \cup B$ where $A$ and $B$ are chosen outside $\Omega^{\prime} \cup \Omega^{\prime \prime}$ and the measure $\mu$ is extended in such a way that $\mu\left(A_{n+1}\right)=\mu\left(B_{n+1}\right)=\tilde{S}_{n}(k+y, x)$. Depending on the sign of $k+y$ we have either $\mu(A)=0$ or $\mu(B)=0$, i.e. we may choose $A=\emptyset$ or $B=\emptyset$. We will show that the so defined collections $A_{i}$ and $B_{i}$ satisfy (1b), (2b) and (3b) for $n+1$. Having done this, we can conclude that $\mu\left(\bigcup_{i=1}^{n+1} B_{i}\right) \geq S_{n+1}(k, x)$ and then the proof can be finished as follows:

$$
\begin{aligned}
\tilde{S}_{n+1}(k, x) \leq \mu\left(\bigcup_{i=1}^{n+1} B_{i}\right)=\mu\left(\bigcup_{i=1}^{n} B_{i}^{\prime}\right)+\mu\left(B_{n+1}\right) & \\
& =\tilde{S}_{n}(k, x+y)+\tilde{S}_{n}(k+y, x) .
\end{aligned}
$$


The properties (2b) and (3b) are easy to see. In fact we have

$$
\begin{aligned}
\mu\left(\bigcup_{i=1}^{n+1} A_{i}\right)+k & =\mu\left(\bigcup_{i=1}^{n} A_{i}^{\prime}\right)+\mu\left(A_{n+1}\right)+k \\
& =\mu\left(\bigcup_{i=1}^{n} B_{i}^{\prime}\right)+\mu\left(B_{n+1}\right)=\mu\left(\bigcup_{i=1}^{n+1} B_{i}\right)
\end{aligned}
$$

and

$$
\mu\left(\bigcap_{i=1}^{n+1} A_{i}\right)=\mu\left(\bigcap_{i=1}^{n} A_{i}^{\prime \prime}\right)=\mu\left(\bigcap_{i=1}^{n} B_{i}^{\prime \prime}\right)+(-1)^{n} x=\mu\left(\bigcap_{i=1}^{n+1} B_{i}\right)-(-1)^{n+1} x .
$$

To see (1b) first notice that by construction we have

$$
\mu\left(\bigcap_{i \in S} A_{i}\right)=\mu\left(\bigcap_{i \in S} B_{i}\right)
$$

whenever $|S| \leq n-2$ or $|S|=n-1$ and $n+1 \in S$. The only difficult part is to prove the equality when $|S|=n-1$ and $n+1 \notin S$. In that case we have

$$
\begin{aligned}
\mu\left(\bigcap_{i \in S} A_{i}\right) & =\mu\left(\bigcap_{i \in S} A_{i}^{\prime}\right)+\mu\left(\bigcap_{i \in S} A_{i}^{\prime \prime}\right) \\
& =\left[\mu\left(\bigcap_{i \in S} B_{i}^{\prime}\right)+\frac{k+(x+y)}{n}\right]+\left[\mu\left(\bigcap_{i \in S} B_{i}^{\prime \prime}\right)-\frac{(k+y)+x}{n}\right] \\
& =\mu\left(\bigcap_{i \in S} B_{i}\right) .
\end{aligned}
$$

This finishes the proof of (1b).

\subsection{Solving the recursion formula}

In this section we derive an explicit formula for the recursion formula in Theorem 3.4. To that end we define the numbers

$$
c_{n, i}:=2^{n-2}-\sum_{j=0}^{i-1}\left(\begin{array}{c}
n-1 \\
j
\end{array}\right), \quad-1 \leq i \leq n
$$

(so $c_{-1}=c_{0}=2^{n-2}$ ), and the intervals

$$
\begin{aligned}
I_{n,-1} & :=(-\infty, 0] \\
I_{n, j} & :=\left[\frac{j}{n-j}, \frac{j+1}{n-j-1}\right] \quad(0 \leq j \leq n-2) \\
I_{n, n-1} & :=[n-1, \infty) .
\end{aligned}
$$




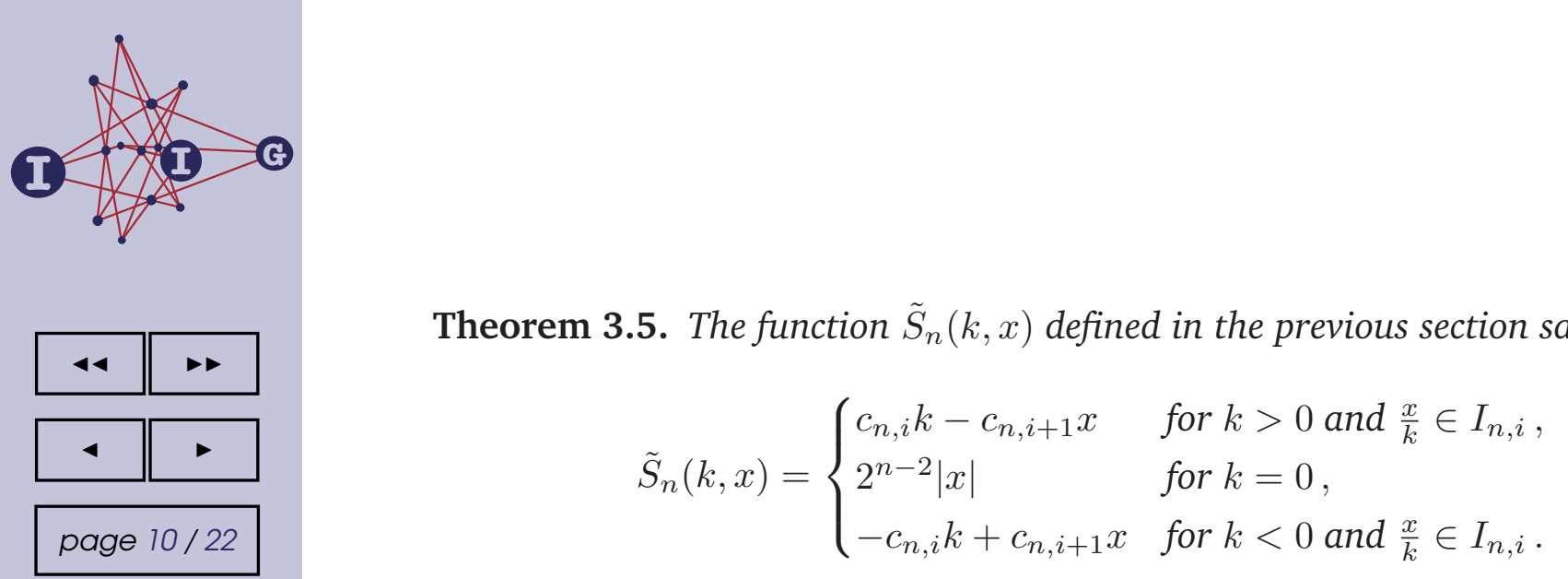

Theorem 3.5. The function $\tilde{S}_{n}(k, x)$ defined in the previous section satisfies

$$
\tilde{S}_{n}(k, x)= \begin{cases}c_{n, i} k-c_{n, i+1} x & \text { for } k>0 \text { and } \frac{x}{k} \in I_{n, i} \\ 2^{n-2}|x| & \text { for } k=0 \\ -c_{n, i} k+c_{n, i+1} x & \text { for } k<0 \text { and } \frac{x}{k} \in I_{n, i}\end{cases}
$$

Proof. We may restrict ourselves to $k \geq 0$ since $\tilde{S}_{n}(-k, x)=\tilde{S}_{n}(k,-x)$ by definition. First note that

$$
c_{n, i}-c_{n, i+1} \frac{i+1}{n-i-1}=c_{n, i+1}-c_{n, i+2} \frac{i+1}{n-i-1}
$$

and thus the function on the right hande side of (6) is well-defined. We prove (6) using induction on $n \geq 2$.

To determine $\tilde{S}_{2}(k, x)$, we search sets $A_{1}, A_{2}, B_{1}, B_{2}$ with $\mu\left(A_{1} \cap A_{2}\right)+x=$ $\mu\left(B_{1} \cap B_{2}\right)$ and $\mu\left(A_{1} \cup A_{2}\right)+k=\mu\left(B_{1} \cup B_{2}\right)$. It is easy to see that this implies

$$
\mu\left(B_{1} \cup B_{2}\right) \geq \max \{x, k\} .
$$

Also, if $x<0$, then $\mu\left(A_{1} \cup A_{2}\right) \geq \mu\left(A_{1} \cap A_{2}\right) \geq-x$ and thus $\mu\left(B_{1} \cup B_{2}\right) \geq k-x$. Moreover, equality can be obtained easily. For example, if $x>k \geq 0$, choose sets satisfying $B_{1}=B_{2}$ and $A_{2}=\emptyset$ and such that $\mu\left(B_{i}\right)=x, \mu\left(A_{1}\right)=x-k$ and $\mu\left(A_{2}\right)=0$. Thus for $k \geq 0$ we have

$$
\tilde{S}_{2}(k, x)= \begin{cases}k-x & \text { for } x \leq 0 \\ k & \text { for } 0 \leq x \leq k \\ x & \text { for } k \leq x\end{cases}
$$

which proves (6) for $n=2$.

For the induction step assume now that $n \geq 3$. If $k=0$ we may assume without loss of generality $x \geq 0$. In this case the recursion formula (3) gives

$$
\begin{aligned}
\tilde{S}_{n+1}(0, x) & =\tilde{S}_{n}\left(0, \frac{n}{n+1} x\right)+\tilde{S}_{n}\left(-\frac{x}{n+1}, x\right) \\
& =2^{n-2} \frac{n}{n+1} x+\left(c_{n,-1} \frac{x}{n+1}+c_{n, 0} x\right) \\
& =2^{n-1} x
\end{aligned}
$$

as desired. Finally consider the case when $n \geq 3$ and $k>0$. Put $y=-\frac{k+x}{n+1}$. Using

$$
\frac{x+y}{k}=\frac{n}{n+1} \cdot \frac{x}{k}-\frac{1}{n+1} \text { and, for } x \neq n k, \frac{x}{k+y}=\frac{n+1}{n \frac{k}{x}-1},
$$



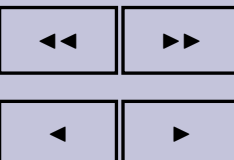

page $11 / 22$

go back

full screen

close

quit

it is straightforward to check the following implications.

$$
\begin{aligned}
\frac{x}{k} \in I_{n+1, j} & \Rightarrow \frac{x+y}{k} \in I_{n, j-1} \text { for } 0 \leq j \leq n ; \\
\frac{x}{k} \in I_{n+1,-1} & \Rightarrow \frac{x+y}{k} \in I_{n,-1} ; \\
n \neq \frac{x}{k} \in I_{n+1, j} & \Rightarrow \frac{x}{k+y} \in I_{n, j} \quad \text { for }-1 \leq j \leq n-1 ; \\
\frac{x}{k}=n & \Rightarrow k+y=0 ; \\
\frac{x}{k}>n & \Rightarrow k+y<0 \text { and } \frac{x}{k+y} \in I_{n,-1} .
\end{aligned}
$$

Hence, for $0 \leq j \leq n-1$ and $\frac{x}{k} \in I_{n+1, j}$ we have

$$
\begin{aligned}
\tilde{S}_{n+1}(k, x) & =\tilde{S}_{n}(k, x+y)+\tilde{S}_{n}(k+y, x) \\
& =\left[c_{n, j-1} k-c_{n, j}(x+y)\right]+\left[c_{n, j}(k+y)-c_{n, j+1} x\right] \\
& =c_{n+1, j} k-c_{n, j+1} x,
\end{aligned}
$$

since $c_{n, j-1}+c_{n, j}=c_{n+1, j}$ by the recursion formula of the binomial coefficients. If $\frac{x}{k} \in I_{n+1,-1}$ we have

$$
\begin{aligned}
\tilde{S}_{n+1}(k, x) & =\tilde{S}_{n}(k, x+y)+\tilde{S}_{n}(k+y, x) \\
& =\left[2^{n-2} k-2^{n-2}(x+y)\right]+\left[2^{n-2}(k+y)-2^{n-2} x\right] \\
& =2^{n-1} k-2^{n-1} x .
\end{aligned}
$$

And finally for $\frac{x}{k} \in I_{n+1, n}$ we have $k+y \leq 0$ and thus

$$
\begin{aligned}
\tilde{S}_{n+1}(k, x) & =\tilde{S}_{n}(k, x+y)+\tilde{S}_{n}(k+y, x) \\
& =\left[\left(1-2^{n-2}\right) k+2^{n-2}(x+y)\right]+\left[-2^{n-2}(k+y)+2^{n-2} x\right] \\
& =\left(-2^{n-1}+1\right) k+2^{n-1} x .
\end{aligned}
$$

This proves the formula for $\tilde{S}_{n+1}$ and completes the induction.

With the explicit formula we are able to determine $\min _{x} \tilde{S}_{n}(k, x)$.

\section{Theorem 3.6.}

$$
\min \left\{\frac{\tilde{S}_{n}(k, x)}{k} \mid k, x \in \mathbb{R}, k>0\right\}=\left(\begin{array}{c}
n-1 \\
\left\lfloor\frac{n-1}{2}\right\rfloor
\end{array}\right) .
$$






$$
\left|\bigcap_{i \in S} B_{i} \backslash \bigcup_{i \notin S} B_{i}\right|= \begin{cases}|S|-\left\lceil\frac{n}{2}\right\rceil & \text { if }|S|>n / 2, \text { and }|S| \text { is even } \\ \left\lceil\frac{n}{2}\right\rceil-|S| & \text { if }|S|<n / 2, \text { and }|S| \text { is odd } \\ 0 & \text { in all other cases }\end{cases}
$$

Theorem 3.8. For each positive integer $n$, the collections $A_{1}, \ldots, A_{n}$ and $B_{1}, \ldots, B_{n}$ described above satisfy

$$
\begin{aligned}
\left|B_{1} \cup \cdots \cup B_{n}\right| & =\frac{n}{2}\left(\begin{array}{c}
n-1 \\
\left\lfloor\frac{n-1}{2}\right\rfloor
\end{array}\right) \\
\left|B_{1} \cup \cdots \cup B_{n-1}\right| & =\left|A_{1} \cup \cdots \cup A_{n-1}\right|+1
\end{aligned}
$$

and

$$
\left|B_{1} \cup \cdots \cup B_{n-k}\right|=\left|A_{1} \cup \cdots \cup A_{n-k}\right| \text { for each } k \geq 2 \text {. }
$$

Proof. To simplify notation, we shall prove this only for $n=4 m$. The other cases can be handled by similar arguments. In the proof we will make use of the following well known identities:

$$
\begin{array}{ll}
\sum_{i=0}^{n}(-1)^{i}\left(\begin{array}{l}
n \\
i
\end{array}\right)=0 & \text { for } n \geq 1, \\
\sum_{i=0}^{n}(-1)^{i} i\left(\begin{array}{l}
n \\
i
\end{array}\right)=0 & \text { for } n \geq 2 .
\end{array}
$$

(The second identity follows from the first using $i\left(\begin{array}{c}n \\ i\end{array}\right)=n\left(\begin{array}{c}n-1 \\ i-1\end{array}\right)$.) We have

$$
\left|\bigcap_{i \in S} A_{i}\right|=\sum_{S \subseteq S^{\prime} \subseteq\{1, \ldots, n\}}\left|\bigcap_{i \in S^{\prime}} A_{i} \backslash \bigcup_{i \notin S^{\prime}} A_{i}\right|
$$

and thus

$$
\left|\bigcap_{i=1}^{n-k} B_{i}\right|-\left|\bigcap_{i=1}^{n-k} A_{i}\right|=\sum_{i=0}^{k}(-1)^{i}(2 m-i)\left(\begin{array}{c}
k \\
i
\end{array}\right) ;
$$

by (15) and (16) this sum vanishes for $k \geq 2$. With the inclusion-exclusion 





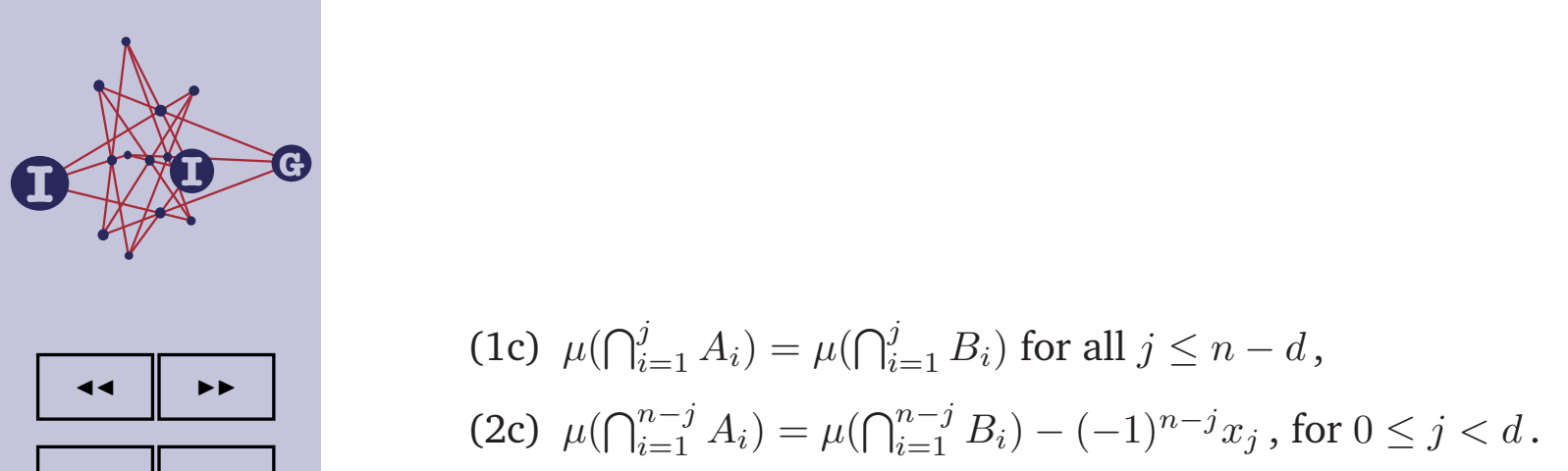

As in the previous sections we ask for the smallest possible value $S_{n}\left(x_{0}, \ldots, x_{d-1}\right)$ for $\max \left\{\mu\left(\bigcup_{i=1}^{n} A_{i}\right), \mu\left(\bigcup_{i=1}^{n} B_{i}\right)\right\}$ where again the minimum is taken over all collections of sets in all finite measurable spaces. With arguments similar to Theorem 3.4 we obtain the following result.

Theorem 4.1. The function $S_{n}\left(x_{0}, \ldots, x_{d-1}\right)$ defined above satisfies the recursion formula

$$
\begin{aligned}
S_{n+1}\left(x_{0}, \ldots, x_{d-1}\right) \geq S_{n}\left(x_{0}+x_{1}, x_{1}+x_{2}, \ldots, x_{d-2}\right. & \left.+x_{d-1}, x_{d-1}+0\right) \\
& +S_{n}\left(x_{0}, \ldots, x_{d-1}\right)
\end{aligned}
$$

for $n \geq d$.

Proof. As in the proof of Lemma 3.1 we define $A_{i}^{\prime}=A_{i} \backslash A_{n+1}, B_{i}^{\prime}=B_{i} \backslash B_{n+1}$, $A_{i}^{\prime \prime}=A_{i} \cap A_{i+1}$ and $B_{i}^{\prime \prime}=B_{i} \cap B_{n+1}$.

It is easy to check intersections of collections $A_{i}^{\prime \prime}, B_{i}^{\prime \prime}$ to see that

$$
\max \left\{\mu\left(\bigcup_{i=1}^{n} A_{i}^{\prime \prime}\right), \mu\left(\bigcup_{i=1}^{n} B_{i}^{\prime \prime}\right)\right\} \geq S_{n}\left(x_{0}, \ldots, x_{d-1}\right) .
$$

And it is only slightly more complex the see that

$$
\begin{aligned}
\mu\left(\bigcap_{i=1}^{n-j} A_{i}^{\prime}\right)= & \mu\left(\bigcap_{i=1}^{n-1} A_{i}\right)-\mu\left(\bigcap_{i=1}^{n-1} A_{i} \cap A_{n+1}\right) \\
= & {\left[\mu\left(\bigcap_{i=1}^{n-1} B_{i}\right)-(-1)^{n+1-j} x_{j}\right] } \\
& \quad-\left[\mu\left(\bigcap_{i=1}^{n-1} B_{i} \cap B_{n+1}\right)-(-1)^{n+1-j-1} x_{j+1}\right] \\
= & \mu\left(\bigcap_{i=1}^{n-j} B_{i}^{\prime}\right)-(-1)^{n-j}\left(-x_{j}-x_{j+1}\right)
\end{aligned}
$$

where $x_{j}=0$ for $j \geq d$. Thus

$$
\begin{aligned}
\max \left\{\mu\left(\bigcup_{i=1}^{n} A_{i}^{\prime}\right), \mu\left(\bigcup_{i=1}^{n} B_{i}^{\prime}\right)\right\} & \\
& \geq S_{n}\left(x_{0}+x_{1}, x_{1}+x_{2}, \ldots, x_{d-2}+x_{d-1}, x_{d-1}+0\right) .
\end{aligned}
$$




$$
\begin{aligned}
& 1 \\
& S_{n+1}\left(x_{0}, \ldots, x_{d-1}\right) \\
& =\max \left\{\mu\left(\bigcup_{i=1}^{n} A_{i}\right), \mu\left(\bigcup_{i=1}^{n} B_{i}\right)\right\} \\
& =\max \left\{\mu\left(\bigcup_{i=1}^{n} A_{i}^{\prime}\right), \mu\left(\bigcup_{i=1}^{n} B_{i}^{\prime}\right)\right\}+\mu\left(A_{n+1}\right) \\
& \geq \max \left\{\mu\left(\bigcup_{i=1}^{n} A_{i}^{\prime}\right), \mu\left(\bigcup_{i=1}^{n} B_{i}^{\prime}\right)\right\}+\max \left\{\mu\left(\bigcup_{i=1}^{n} A_{i}^{\prime \prime}\right), \mu\left(\bigcup_{i=1}^{n} B_{i}^{\prime \prime}\right)\right\} \\
& =S_{n}\left(x_{0}+x_{1}, x_{1}+x_{2}, \ldots, x_{d-2}+x_{d-1}, x_{d-1}+0\right)+S_{n}\left(x_{0}, \ldots, x_{d-1}\right) \text {, }
\end{aligned}
$$
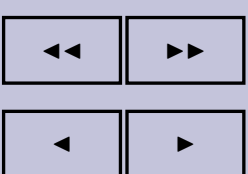

quit

which completes the proof.

With the trivial bound $S_{d-1}\left(x_{0}, \ldots, x_{d-1}\right) \geq\left|x_{d-1}\right|$ we can use Theorem 4.1 to obtain

$$
S_{n}\left(x_{0}, \ldots, x_{d-1}\right) \geq 2^{n-d+1}\left|x_{d-1}\right| .
$$

This is almost all we need for the following asymptotic theorem.

Theorem 4.2. Let $A_{1}, \ldots, A_{n}$ and $B_{1}, \ldots, B_{n}$ be two collections of sets satisfying $\left|\bigcap_{i \in S} A_{i}\right|=\left|\bigcap_{i \in S} B_{i}\right|$ for all subsets $S \subseteq\{1, \ldots, n\}$ with $|S| \leq n-d$. Then

$$
\frac{\left|\bigcup_{i=1}^{n} B_{i}\right|-\left|\bigcup_{i=1}^{n} A_{i}\right|}{\left|\bigcup_{i=1}^{n} B_{i}\right|} \leq O\left(n^{d-1} 2^{-n}\right)
$$

or equivalently

$$
\frac{\left|\bigcup_{i=1}^{n} A_{i}\right|}{\left|\bigcup_{i=1}^{n} B_{i}\right|} \leq 1+O\left(n^{d-1} 2^{-n}\right) .
$$

Remarks. (1) Remember that we assume $d$ constant in this section. The $O$-constant in the theorem will thus depend on $d$.

(2) This bound is much better than the one given in [2, Theorem 1, Part 2].

(3) This a remark that motivates the method used in the proof. Clearly

$$
\frac{\mu\left(\bigcup_{i=1}^{n} B_{i}\right)-\mu\left(\bigcup_{i=1}^{n} A_{i}\right)}{\mu\left(\bigcup_{i=1}^{n} B_{i}\right)}=\frac{\sum_{i=0}^{d-1}\left(\begin{array}{c}
n \\
i
\end{array}\right) x_{i}}{S_{n}\left(x_{0}, \ldots, x_{d-1}\right)} .
$$

If we could assure that $\left|x_{i}\right| \leq c n^{d-1-i}\left|x_{d-1}\right|$ for some constant $c$, then the 
theorem would follow directly from

$$
\begin{aligned}
& \frac{\sum_{i=0}^{d-1}\left(\begin{array}{c}
n \\
i
\end{array}\right) x_{i}}{S_{n}\left(x_{0}, \ldots, x_{d-1}\right)} \leq \frac{\sum_{i=0}^{d-1}\left(\begin{array}{c}
n \\
i
\end{array}\right) n^{d-1-i} c x_{d-1}}{S_{n}\left(x_{0}, \ldots, x_{d-1}\right)} \\
& =\frac{O\left(n^{d-1}\left|x_{d-1}\right|\right)}{\left.2^{n-d+1}\left|x_{d-1}\right|\right)}=O\left(n^{d-1} 2^{-n}\right) .
\end{aligned}
$$

go back

full screen

close

quit

The difficulty of the proof is to remove the restriction $\left|x_{i}\right| \leq c n^{d-1-i}\left|x_{d-1}\right|$.

Proof of Theorem 4.2. Choose constants $c_{0}, \ldots, c_{d-1}>0$ satisfying

$$
1-\sum_{j=i+1}^{d-1}\left(\begin{array}{c}
n-d-1 \\
j-i
\end{array}\right) \frac{c_{i}}{c_{j} n^{j-i}} \geq \frac{1}{2}
$$

for $i=0, \ldots, d-2$. Let $i_{\max }$ be the value of $i$ for which $c_{i} n^{i}\left|x_{i}\right|$ is maximal. Using an inductive argument, the recursion formula (19) shows that

$$
S_{n}\left(x_{0}, \ldots, x_{d-1}\right) \geq \sum_{j=1}^{n+1-d}\left(\begin{array}{c}
n+1-d \\
j
\end{array}\right) S_{d-1}\left(x_{0}^{(j)}, \ldots, x_{d-1}^{(j)}\right)
$$

where

$$
x_{k}^{(j)}=\sum_{\ell=k}^{d-1}\left(\begin{array}{c}
j \\
\ell-k
\end{array}\right) x_{\ell} .
$$

For $k=i_{\max }$ and all $j \in\{1, \ldots, n-d+1\}$ this implies that (in the second step we use the definition of $i_{\max }$ and the last step uses (20))

$$
\begin{aligned}
\left|x_{i_{\max }}^{(j)}\right| & \geq\left|x_{i_{\max }}\right|-\sum_{\ell=i_{\max }+1}^{d-1}\left(\begin{array}{c}
n-d+1 \\
\ell-i_{\max }
\end{array}\right)\left|x_{\ell}\right| \\
& \geq\left|x_{i_{\max }}\right|-\sum_{\ell=i_{\max }+1}^{d-1}\left(\begin{array}{c}
n-d-1 \\
\ell-i_{\max }
\end{array}\right) \frac{c_{i_{\max }}}{c_{\ell} n^{\ell-i_{\max }}}\left|x_{i_{\max }}\right| \\
& \geq \frac{1}{2}\left|x_{i_{\max }}\right| .
\end{aligned}
$$

Together with the trivial bound

$$
S_{d-1}\left(x_{0}^{(n-d+1, i)}, \ldots, x_{d-1}^{(j)}\right) \geq\left|x_{i_{\max }^{(j)}}\right|
$$

and (21) we find

$$
S_{n}\left(x_{0}, \ldots, x_{d-1}\right) \geq \sum_{j=1}^{n+1-d}\left(\begin{array}{c}
n+1-d \\
j
\end{array}\right) \frac{1}{2}\left|x_{i_{\max }}\right|=2^{n-d}\left|x_{i_{\max }}\right| .
$$




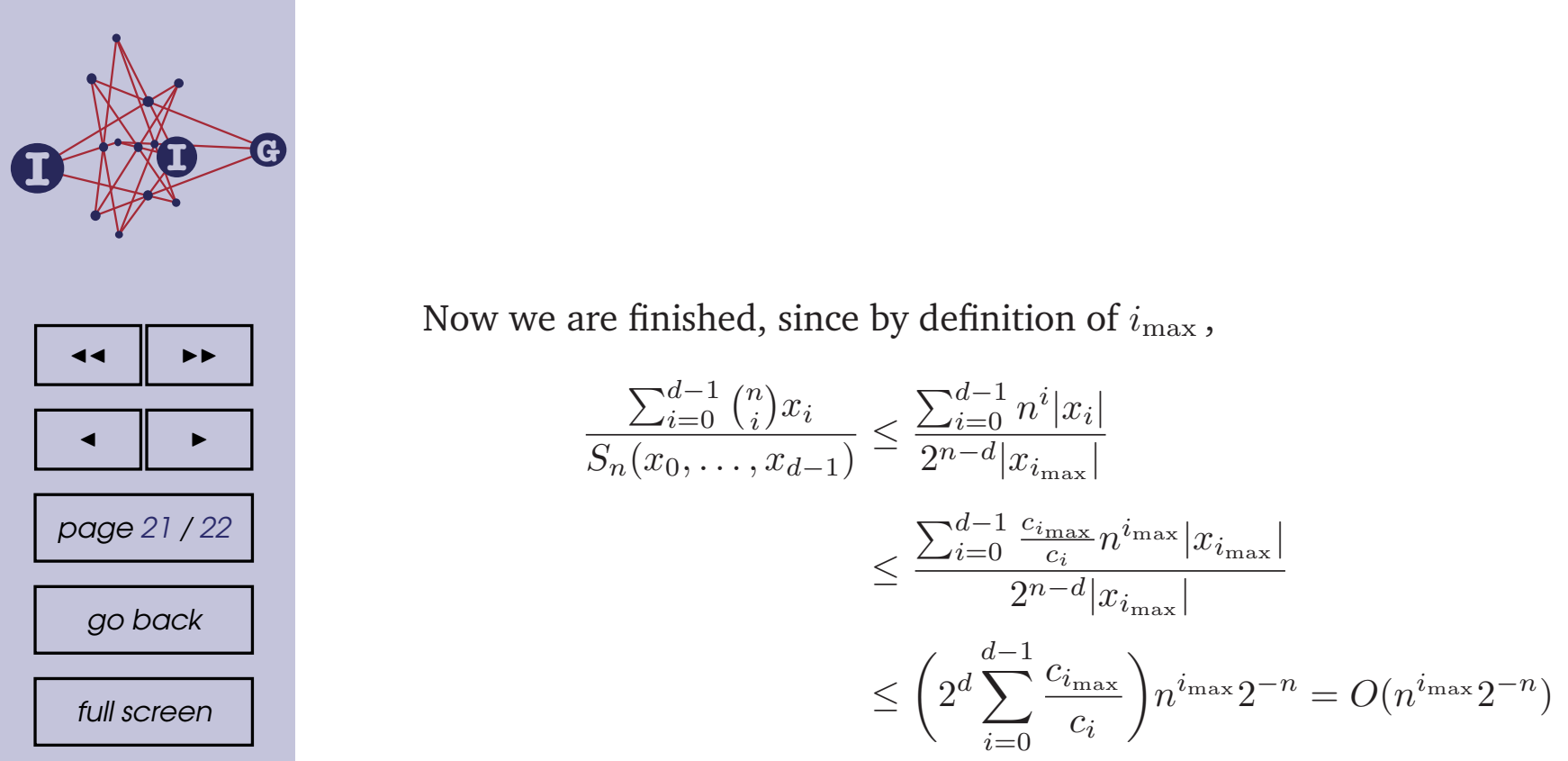

The worst bound is obtained for $i_{\max }=d-1$, i.e. in the simple case $\left|x_{i}\right| \leq$ $c n^{d-1-i}\left|x_{d-1}\right|$ mentioned in Remark (3) above.

\section{Open problems}

We want to close this article with some open problems.

1. What is the discrete analogue for Theorem 3.6, i.e. find the minimal $k$ for which $\min _{x \in \mathbb{Z}} S(k, x)=\min _{x \in \mathbb{R}} \tilde{S}(k, x)$. This is more difficult than the discrete analogue for Theorem 3.7, since this time the discrete minimum has to be asymmetric.

2. The visual cryptography scheme in Theorem 3.10 has randomness $m$ !. That is the trivial upper bound for the randomness. Improve that bound.

3. The explicit solution for $m=n-2$ (Theorem 3.6) proves

$$
\frac{\left|\bigcup_{i=1}^{n} A_{i}\right|}{\left|\bigcup_{i=1}^{n} B_{i}\right|} \leq 1+O\left(\sqrt{n} 2^{-n}\right)
$$

which is better than the general bound

$$
\frac{\left|\bigcup_{i=1}^{n} A_{i}\right|}{\left|\bigcup_{i=1}^{n} B_{i}\right|} \leq 1+O\left(n 2^{-n}\right)
$$

proven in Theorem 4.2. Improve the bound of Theorem 4.2 for $m=n-d$, $d>2$.

4. Is it possible to adapt the arguments of Theorem 4.2 to deal with nonconstant $d$ ? 


\section{References}

[1] J. Kahn, N. Linial and A. Samorodnitsky, Inclusion-exclusion: exact and approximate, Combinatorica 16, no. 4 (1996), 465-477.

[2] N. Linial and N. Nisan, Approximate inclusion-exclusion, Combinatorica 10, no. 4 (1990), 349-365.

[3] M. Naor and A. Shamir, Visual cryptography, in Alfredo De Santis, editor, Advances in cryptology - EUROCRYPT '94, Lect. Notes Comput. Sci. 950, 1-12, Springer-Verlag, 1995.

close

quit

Andreas Klein

Ghent University, Dept. of Pure Mathematics and Computer Algebra, KrijgslaAn 281S22, 9000 GHENT, BELGIUM

e-mail: klein@cage. ugent.be

Klaus Metsch

Mathematisches Institut, Arndtstrasse 2, D-35392 Giessen, Germany

e-mail: klaus.metsch@math.uni-giessen.de 